\title{
Oral Cancer Screening for Asymptomatic Adults: Do the United States Preventive Services Task Force Draft Guidelines Miss the Proverbial Forest for the Trees?
}

\author{
Paul C Edwards \\ 2013 \\ Oral Surgery, Oral Medicine, Oral Pathology and Oral Radiology \\ 116(2), 131-134
}

As experts in the field and regular readers of this journal, we are all acutely aware of the sobering statistics on oral cancer: in the United States there are an estimated 275,000 men and women alive with a prior diagnosis of oral cavity or pharyngeal cancer ${ }^{1}$. It is estimated that an additional 41,000 cases will be diagnosed in 2013 alone, with just under 8,000 individuals dying of their disease $^{2}$. For the period 2003-2009, the estimated 5 year relative survival rate for patients diagnosed with oral and pharyngeal cancer is $62 \%$ in comparison to the general population ${ }^{1}$. For black men, the results are more discouraging, with a 5 year relative survival of just under $40 \%$. Looking at lifetime risk, a child born today has an estimated $1.1 \%$ likelihood of developing oral cavity or pharyngeal cancer at some point during their life ${ }^{3}$. The stage at which the cancer is diagnosed has a significant effect on overall survival. Localized disease, representing disease confined to the primary site, is associated with an $83 \% 5-$ year survival rate. With spread to the regional lymph nodes, the 5-year survival drops to just under $60 \%$. The statistics are even grimmer in the presence of distant metastasis, with the 5-year survival dropping to $36 \%$.

With that backdrop in mind, the United States Preventive Services Task Force (USPSTF) recently released a draft Recommendation Statement which concluded that for adults age 18 years or older seen in the primary care setting, the "current evidence is insufficient to assess the balance of benefits and harms of screening for oral cancer in asymptomatic adults. Evidence is lacking, of poor quality, or conflicting, and the balance of benefits and harms cannot be determined."

Rather than representing a paradigm shift, this statement is simply an update of the 2004 USPSTF recommendations, which similarly concluded "there was insufficient evidence to recommend for or against routine screening for oral cancer in adults ${ }^{4}$." Other expert groups have reached comparable conclusions. For example, the reader is referred to the "Evidence-based clinical recommendations regarding screening for oral squamous cell carcinomas ${ }^{5 "}$ recently published by a panel convened by the American Dental Association (ADA) Council on Scientific Affairs, which however takes a more pragmatic approach to this question.

Specifically, the USPSTF was unable to offer a recommendation on the benefits versus risks of routine visual oral cancer screening of asymptomatic patients who present in the primary care setting. This decision was based on their assessment of a lack of evidence in three areas: how well do oral cancer screening exams detect disease? Do the harms associated with screening outweigh the potential benefits? Does detection of oral cancer through screening reduce morbidity or mortality?

Accepted Author's Manuscript. Final version published as: 
Rather than arbitrarily denouncing the entirety of these recommendations, the authors are to be at least commended for highlighting the risk factors for developing oral cancer: smoking, heavy alcohol consumption, betel quid use, and, for a subset of oropharyngeal cancers, infection with high risk human papillomavirus virus (although I disagree with the suggestion that there is sufficient evidence at this point to include infection with candida or bacterial flora as oral cancer risk factors). These comments may help to educate both the general public as well as those health care providers who may have less experience in this area. The guidelines also accurately note that potential screening adjuncts, such as toluidine blue, chemiluminescence, autofluorescence and brush cytopathology lack sufficient evidence to recommend their routine use in the primary care setting. In low risk populations, reliance on the results of these screening adjuncts, in the absence of clinical correlation, is associated with an unacceptable rate of false positives. Likewise, the absence of well-designed studies evaluating the risks and benefits of oral cancer screening that are of direct relevance to the population of the United States (the target audience of this draft statement) must be acknowledged.

However, an alternative approach to looking at this question should be considered, one that also requires examining this issue from a clinical perspective (curiously, there is no indication that the USPSTF sought input from clinical specialists in this area; namely oral pathologists, oral surgeons, general dentists, public health dentists, etc...). Let me clarify, before being misquoted, that I am not implying that biomedical science and clinical care are opposing paradigms. We are all acutely aware that, as health care providers, treatment decisions must be based on a solid evidence-based foundation backed by rigorous scientific investigation. Rather, the approach that should be followed in answering this question is one that reconciles the available evidence, or lack thereof, with a more clinically applicable approach that takes into account the realities of the primary care dental setting. With that in mind, lets review these areas:

1. How well do oral cancer screening exams detect disease (i.e. their performance characteristics)?

For the purpose of this discussion, the screening examination should be defined as a thorough visual and tactile inspection of the head and neck structures and the accessible oral cavity executed by a well-trained general dentist in the primary care dental setting, and performed on all patients of record as part of the routine patient assessment process ${ }^{6}$. As needed, this is supplemented by biopsies of any areas of suspicion, with the goal being to identify all variations from normal, including but not restricted to potentially preneoplastic conditions (I prefer this term over the WHO's terminology of "potentially malignant disorders") and early stage oral cancer. 
As noted in the USPSTF report, the two studies that most approximate these characteristics are from the United Kingdom, which has an oral cancer prevalence similar to that of the United States. These studies reported sensitivities in the low 70s and specificities approaching 100 percent, although these also highlighted the dilemma of identifying the gold standard, which in these studies was a second examination by a specialist in oral pathology/oral medicine.

Examining this from a more pragmatic perspective, considering that greater than $90 \%$ of intraoral malignancies are represented by squamous cell carcinomas, a neoplasm of surface epithelium, a thorough visual and tactile examination by a well-trained dentist in the primary care setting, coupled with a reasonable degree of suspicion for all white, red or ulcerated lesions of undetermined etiology, should permit identification of the vast majority of early oral squamous cell carcinomas. Clearly, the accuracy with which dental providers in the primary care setting can identify and triage potentially preneoplastic conditions and early stage oral cancer is dependent on both their training and clinical experience.

Consequently, as educators, it is critical that our dental school graduates are both proficient and confident in their ability to assess soft tissue lesions. Additionally, as suggested in the recent guidelines from the ADA's Council on Scientific Affairs referred to previously, "the clinician can reduce the risk of performing unnecessary biopsies by obtaining an opinion by a dental or medical care provider who has advanced training and experience in diagnosis of oral cancer and its precursor lesions 5 ."

More importantly though, these guidelines fail to recognize that it is not realistic to separate the oral cancer screening component from the overall comprehensive head and neck examination that all primary care dental providers perform on their patients. This intraoral and extraoral examination is arguably the most important component of every dental patient's routine assessment, and includes a thorough review not just of the teeth and periodontal supporting structures, but of all hard and soft tissues of the visible oral cavity, as well as the cervical area and the skin of the face. The purpose of this examination is to identify every departure from normal, ranging from the more common tooth-related conditions, such as abscessed teeth and periodontal disease, to infectious processes (e.g. candidiasis; oral manifestations of HIV infection; deep fungal infections), reactive soft tissue lesions (e.g. mucoceles, fibromas), and immune-mediated processes (e.g. lichen planus, pemphigoid), to name a few. The diagnosis and management of these non-malignant processes is a critical component of the day-to-day practice of dentistry, ${ }^{7}$ and consequently the identification of these conditions cannot be arbitrarily separated from the "oral cancer" screening exam.

2. Do the harms associated with screening outweigh the potential benefits? The draft version of the USPSTF report states that "none of the studies in our review reported on harms from the screening test itself or from false-positive or 
false-negative test results. Screening using visual inspection and palpation should be low risk. However, any time devoted to it would reduce opportunity for other interventions that might have greater impact on health outcomes."

In reality, a thorough head and neck examination by a well-trained and competent dentist in the primary care setting requires no special equipment (good lighting, a dental mirror and gauze), no additional expense, and at most 23 minutes of the practitioner's time. Other than the minor potential surgical risks associated with a biopsy procedure, where deemed necessary, routine oral screening is in no way an invasive, time consuming, or costly procedure, and is not associated with any significant degree of potential intraprocedural morbidity (e.g. perforation following colonoscopy for the detection of colorectal cancer) or long-term sequelae resulting from exposure to ionizing radiation (e.g. mammography for the detection of breast cancer).

It is also worth pointing out that the potential benefits of performing an oral cancer screening examination are not simply limited to detecting patients with oral cancer or potentially preneoplastic conditions. In addition to the many more commonly encountered conditions that are identified by means of the examination, this is also an opportunity to start a dialogue between clinician and patient on aspects such as modifying risk factors that are associated with an increased risk of developing oral cancer. As practitioners, we have all experienced the patient who, after undergoing a routine oral cancer examination, volunteers that he or she is interested in quitting smoking. There is also evidence that patients with potentially preneoplastic conditions of the oral cavity, such as idiopathic leukoplakia, may benefit from the elimination of risk factors such as smoking ${ }^{8}$.

It is also accepted that many potentially preneoplastic conditions of the oral cavity, such as verrucous hyperplasia, have an unpredictable natural course, and overtreatment could result in adverse consequences for the patient. But rather than discount the potential benefits of early detection in these situations, would it not be better to instead develop clinically relevant guidelines for the assessment and management of patients identified with these conditions that would help guide our colleagues in the primary care setting?

Ultimately however, the balance as to whether the potential benefits of routinely screening for oral cancer outweigh the risks depends on the answer to the next question:

3. Does detection of oral cancer through screening reduce morbidity or mortality? As with other position papers in this area, the USPSTF statement bases much of its analysis on the findings from the Trivandrum Oral Cancer Screening Study ${ }^{9}$, in which administrative units in the Trivandrum district, an area of India known to have a high risk of oral cancer, were randomized so that residents either received three rounds of home-based oral screening at 3-year intervals by non- 
medical university graduates with minimal training in oral pathology or were assigned to a non-intervention control group. The overall mortality rate from oral cancer did not differ between the groups in the screened districts versus those in the control districts. However, participants from the intervention group who smoked or used alcohol had significantly lower overall mortality rates from oral cancer than participants from the unscreened control group. Among subjects who did not use tobacco or alcohol, these differences were not significant. While not intending to critique the methodology of this study, there are recognized differences in study parameters that preclude directly extrapolating from these findings to the U.S. situation: the incidence of oral cancer is much higher in the geographic area studied than in the U.S., tobacco use in the study area consists predominantly of bidi, a locally produced cigarette associated with a potentially greater risk of oral cancer development than conventional cigarettes ${ }^{10}$, minimally trained non-health care workers performed the screening examinations, the study involved home visits to the study participants with the explicit goal of performing an oral cancer examination as opposed to being a limited part of a routine dental examination and only $62 \%$ of study participants with identified lesions complied with referral for follow up treatment. Interestingly though, patients identified with oral cancer in the intervention group were more likely to have early stage disease (41\% versus $23 \%$ stage I or II) and higher 5 -year survival rate (50\% versus $34 \%$ ) compared with the control group.

This segues nicely to another issue posed by the USPSTF, namely the uncertainty regarding how much of the improved survival associated with early diagnosis is due to lead time bias (implying that an earlier diagnosis leads to an apparent longer survival regardless of whether the earlier access to treatment affects the natural history of the disease), or length time bias (the observation that because there are subsets of oral cancers that progress at different rates, those that progress more rapidly than others, and hence lead to earlier death, are less likely to be detected by routine screening; the inference being that those cancers that are detected at an earlier stage through screening examinations may inherently have less aggressive biologic potential, thereby making it appear that early diagnosis leads to improved survival). The USPSTF draft statement argues that "harms of treatment for screen-detected oral cancer and its potential precursors ... may result from complications of surgery, radiotherapy, and chemotherapy. The natural history of screen-detected oral cancer is not well understood and, as a result, the harms from overdiagnosis and overtreatment are not known".

There is no doubt, as alluded to above, that tumor growth rates vary, based on factors such as the inherent biologic properties of the tumor, host resistance and anatomic location. But how does this "limited knowledge" of the natural history of screen-detected oral cancer translate to the clinical setting? As a clinician, upon diagnosing an early stage cancer in an otherwise healthy patient, would you ever consider advising your patient that the benefits of undergoing treatment are unknown, since "the natural history of screen-detected oral cancer is not well 
understood and ... the harms from ... overtreatment are not known"?

The implication that we should not perform routine screenings in the primary care setting is clearly not in our patients' best interests. Not attempting to identify cancerous lesions at their earliest stage, particularly when this can be accomplished by means of a simple non-invasive examination while the patient is already sitting in the dental chair, could be viewed as paternalistic. While there clearly can be indications for not proceeding with treatment for a small subset of patients diagnosed with oral cancer through screening, e.g. due to poor overall health status, even in these cases, this decision should only be made following a dialogue that recognizes the patient's wishes, the likelihood of a favorable outcome and the recommendations of the treatment team (i.e. informed consent). Ultimately, in order for the patient to make an informed decision, does the cancer not have to first be identified?

In summary, several questions come to mind after reading these guidelines. Why the arbitrary attempt to separate the "oral cancer screening" from the overall head and neck examination? What guidance, if any, do these recommendations offer to the patient or the practicing clinician? Do the inevitable news headlines suggesting that there is no clear benefit to screening for oral cancer send the right message at this point in time, considering the progress that has been made in reducing the death rate from oral cancer over the past several decades? Does the emphasis on the absolute need for suitably powered randomized controlled trials conclusively supporting the benefit of routine oral cancer screening in the primary care setting ultimately benefit our patients? By not performing a routine oral cancer examination as part of a comprehensive overall oral health assessment, what impact does this have on potential "teachable moments" with our patients? Finally, if primary care dental providers, who have the depth and breadth of knowledge to identify which oral lesions are potentially worrisome and which are not, don't provide this crucial service, who will?

I leave the reader with the following insightful comments from Dr. Robert $A$. Faiella, president of the American Dental Association (ADA), and Dr. Paul D. Freedman, president of the American Academy of Oral and Maxillofacial Pathology (AAOMP), written in response to similarly worded recommendations published in Consumers Reports magazine ${ }^{11}$ : "Oral cancer screenings are not intended to be a stand-alone or separate procedure ... oral cancer screenings should be part of the complete dental examination that is to be performed on all patients. During these complete exams your dentist is checking for ALL oral diseases and pathologic conditions. These non-invasive visual and tactile examinations, (which are generally included with no additional fee for the cancer screening component) can result in earlier diagnosis of oral cancer specifically but also a multitude of other oral diseases in general. The ADA and AAOMP will continue to support and encourage scientific investigations regarding detection of oral cancer, with the firm belief that one missed oral cancer is one too many ${ }^{12}$." 
${ }^{1}$ From DevCan - Probability of Developing or Dying of Cancer, Surveillance Epidemiology and End Results, National Cancer Institute, U.S. National Institutes of Health. Accessed online (April 30, 2013) at: http://www.seer.cancer.gov/statfacts/html/oralcav.html

${ }^{2}$ From Surveillance Epidemiology and End Results, National Cancer Institute, U.S. National Institutes of Health. Accessed online (April 30, 2013) at: http://www.seer.cancer.gov/csr/1975_2010/results_single/sect_01_table.01.pdf ${ }^{3}$ Data estimated on January 1, 2010, Surveillance Epidemiology and End Results, National Cancer Institute, U.S. National Institutes of Health. Accessed online (April 30, 2013) at http://seer.cancer.gov/csr/1975_2009_pops09/results_merged/topic_lifetime_risk .pdf

${ }^{4}$ U.S. Preventive Services Task Force. Screening for Oral Cancer:

Recommendation Statement. AHRQ Pub. No. 05-0564-A. Rockville, MD: Agency for Healthcare Research and Quality; 2004.

${ }^{5}$ Rethman MP, Carpenter W, Cohen EE, Epstein J, Evans CA, Flaitz CM, Graham FJ, Hujoel PP, Kalmar JR, Koch WM, Lambert PM, Lingen MW, Oettmeier BW Jr, Patton LL, Perkins D, Reid BC, Sciubba JJ, Tomar SL, Wyatt AD Jr, Aravamudhan K, Frantsve-Hawley J, Cleveland JL, Meyer DM; American Dental Association Council on Scientific Affairs Expert Panel on Screening for Oral Squamous Cell Carcinomas. Evidence-based clinical recommendations regarding screening for oral squamous cell carcinomas. J Am Dent Assoc. 2010;141:509-20.

${ }^{6}$ National Institute of Dental and Craniofacial Research. Detecting Oral Cancer: A Guide for Health Care Professionals. Bethesda, MD: National Institutes of Health; 2011. Accessed at http://www.nidcr.nih.gov/OralHealth/Topics/OralCancer/DetectingOralCancer.htm on May 2, 2013.

${ }^{7}$ Bouquot JE. Common oral lesions found during a mass screening examination. J Am Dent Assoc 1986;112:50-7.

${ }^{8}$ Roosaar A, Yin L, Johansson AL, Sandborgh-Englund G, Nyrén O, Axéll T. A long-term follow-up study on the natural course of oral leukoplakia in a Swedish population-based sample. J Oral Pathol Med 2007;36:78-82.

${ }^{9}$ Sankaranarayanan R, Ramadas K, Thomas $\mathrm{G}$, et al. Effect of screening on oral cancer mortality in Kerala, India: a cluster-randomised controlled trial. Lancet 2005;365:1927-33.

${ }^{10}$ Rahman M, Sakamoto J, Fukui T. Bidi smoking and oral cancer: A metaanalysis. International Journal of Cancer 2003;106:600-4.

${ }^{11}$ Save Your Life: 8 Cancer Tests You Need, Plus 3 You Don't. Consumer Reports Magazine, March 2013.

${ }^{12}$ Letter submitted to Consumers Union, March 1, 2013. Personal communication, American Dental Association media relations. 